\title{
Online Bimanual Manipulation Using Surface Electromyography and Incremental Learning
}

\author{
Ilaria Strazzulla, Student Member, IEEE, Markus Nowak, Marco Controzzi, Christian Cipriani, Senior \\ Member, IEEE and Claudio Castellini, Member, IEEE
}

\begin{abstract}
The paradigm of simultaneous and proportional myocontrol of hand prostheses is gaining momentum in the rehabilitation robotics community. As opposed to the traditional surface electromyography classification schema, in simultaneous and proportional control the desired force/torque at each degree of freedom of the hand/wrist is predicted in real-time, giving to the individual a more natural experience, reducing the cognitive effort and improving his dexterity in daily-life activities. In this study we apply such an approach in a realistic manipulation scenario, using ten non-linear incremental regression machines to predict the desired torques for each motor of two robotic hands. The prediction is enforced using two sets of surface electromyography electrodes and an incremental, non-linear machine learning technique called Incremental Ridge Regression with Random Fourier Features. Nine able-bodied subjects were engaged in a functional test with the aim to evaluate the performance of the system. The robotic hands were mounted on two hand/wrist orthopedic splints worn by healthy subjects and controlled online. An average completion rate of more than $95 \%$ was achieved in single-handed tasks and $84 \%$ in bimanual tasks. On average, five minutes of retraining were necessary on a total session duration of about one hour and forty minutes. This work sets a beginning in the study of bimanual manipulation with prostheses and will be carried on through experiments in unilateral and bilateral upper limb amputees thus increasing its scientific value.
\end{abstract}

Index Terms-rehabilitation robotics, machine learning, adaptive systems.

\section{INTRODUCTION}

$\mathrm{R}$ ESTORING motor function of the upper limb after amputation is one of the major challenges in the rehabilitation engineering field. To this aim, researchers have spent a significant amount of effort applying machine learning techniques to the surface electromyography signals (sEMG). The idea of classifying the sEMG patterns to predict the subject's intent and to control an upper limb prosthesis dates back at least to 1967 [1]; it was based on the premise that amputees can voluntarily generate repeatable and distinct EMG signal patterns for each class of motion, which could then be mapped to appropriate prosthesis commands. The technique held the promise to radically advance myocontrol of hand prostheses, with respect to the conventional, two-state amplitude- or rate-modulated controllers (the so called proportional controllers) [2]. However, although significant achievements and results have seen the light in research labs [3],[4], these have impacted the clinical procedures in a very limited manner [5] and recent surveys reveal that abandonment of myo-controlled hand prostheses hovers around 40\% [2].

One of the main drawbacks of conventional classification control schemes is that only a finite number of pre-trained prehensile patterns can be (mutually exclusively) selected, without having the possibility of simultaneous control of multiple functions nor of proportional control. This approach is neither physiologically appropriate nor dexterous because arbitrary movements or hand postures cannot be obtained. In addition, classifiers cannot cope with the changes of EMG signals with time, like sweat, electrode displacement, contact impedance and fatigue [6], if not retrained.

In order to overcome the above mentioned limitations, we hereby follow a different approach called Incremental-Learning Myoelectric Control (ILMC) [7]. We propose a non-linear incremental learning method in which occasional updates with a modest amount of novel training data allow continual adaptation to the changes in the signals. In particular an incremental variant of the Ridge Regression algorithm (iRR) [8] was coupled with Random Fourier Features (RFF) [9] to predict simultaneous and proportional control of multiple degrees of actuation (DoA) of a hand prosthesis. Other approaches aiming at simultaneous and proportional control are based upon Nonnegative Matrix Factorization [10], MultiLayer Perceptron [11], Support Vector Regression [12] or Bayesian theory [13]. These studies focused on wrist movements, rather than on intrinsic hand movements.

The effectiveness of incremental supervised adaptation in myocontrol was demonstrated by Sensinger and colleagues [14], among the others. In their system samples drawn from subsequent training sessions were added incrementally to a baseline model obtained by a Linear Discriminant Classifier; the adaptive system achieved a reduction of the classification error of at least $26 \%$, compared to the non-adaptive one (notice that, in that work, no control over the size of the training set was enforced, which led to high computational requirements). As a further example, Hahne and colleagues demonstrated the advantages of exploiting co-adaptation [15]. In their work, both able-bodied and congenital amputees achieved better myocontrol performance when engaged in a virtual 2D trajectory-following task while the machine learning system was being trained on the same data.

In our previous work we preliminary showed that the ILMC approach was effective for predicting single-finger forces in online tests and for controlling the hand of a humanoid robot to grasp and release everyday-life objects [7]. In the present study, 
we rather use ILMC to have able-bodied subjects control two dexterous robotic hands (Fig. 1). The subjects were engaged in a functional test which involved single-handed or bimanual grasping and manipulation of common objects using the artificial hands, worn on orthopedic splints (Fig. 2), inspired by the Southampton Hand Assessment Procedure (SHAP) [16].

Average success ratios of $\sim 93 \%$ and $\sim 84 \%$ were achieved in single-handed tasks and in bimanual tasks, respectively. To our knowledge this is the first demonstration of bimanual incremental, simultaneous and proportional control of dexterous robotic hands. It represents an advancement both with respect to conventional proportional myoelectric control and to classification, since it enables the individual to control two multi-degree hand prostheses, using a continuous force/posture configuration domain.

The experimental protocol was composed by ten tasks, five for single hand manipulation and five for bimanual manipulation. Each subset of tasks was ordered by increasing difficulty of execution.

The experimental results have been analyzed using statistical tests. One of the goals was to assess the amount of needed incremental retrainings; a second test was conducted to assess the existence of a statistical difference between expert and naïve subjects, while the latter two were performed to confront the subjects' performances in terms of manipulation dexterity: analysis of the completion times (Tcs) relative to intra- and inter-task manipulation. The intra-task manipulation analysis was conducted to assess the preference of the dominant or nondominant hand to the subjects, while the inter-task manipulation was performed to determine a statistical difference between tasks in terms of execution speed.

The experimental results are promising for future clinical applications, especially because bimanual manipulation using two prostheses was never explored so far, to the best of our knowledge; nevertheless, several upper-limb functional assessment protocols, such as, e.g., the aforementioned SHAP and the ACMC [17] include bimanual tasks.

\section{MAteriAls AND MethodS}

\section{A. Participants}

Nine able-bodied subjects with no known history of neuromuscular disorders (eight right-handed, one left-handed, 24 to 42 years old) participated in this study. Three of the participants were expert users (subjects S1, S3, S4 already used the ILMC system and were familiar with the experimental setup) while six were naïve (subjects S2, S5-S9). Informed consent was obtained before the experiment, and formal approval for the experiment was obtained from the Ethical Committee of the DLR.

\section{B. Robotic Hands}

In this work a left- and a right-handed IH2 Azzurra robotic hand (Prensilia S.r.l., Pisa, Italy, Fig. 2) were used. Such hands comprise five DoAs (non-back-drivable electrical motors) allowing for flexion/extension of the thumb, index, middle, coupled ring+little, and rotation of the thumb (hereafter abduction/adduction). Each hand includes encoders on each motor axis, current sensors and low level control loops implementing current, position and torque controls of the DoAs, by receiving reference signals via the serial port.

\section{Incremental-Learning Myoelectric Controller - ILMC}

Incremental Ridge Regression (iRR) with Random Fourier Features (RFFs) was used to convert sEMG signals into control signals for the robotic hands, i.e., to enforce the myoelectric control. iRR is the standard Ridge Regression method (i.e., regularized least-squares optimization [8]) in which rank-1 updates are used to update the model each time a new (sEMG, activation) pair is available. As a rank-1 update method we employed the Sherman-Morrison formula. RFFs are a nonlinear extension to Ridge Regression, first introduced by Rahimi and Recht [18]. iRR with RFFs already proved to be effective in myoelectric control of dexterous prosthetic hands [7]; we hereby sketch their mathematical foundations without giving the details. The interested reader is referred to [7].

The input space is represented by $d$-dimensional sEMG signals $\boldsymbol{e}$ directly gathered from the electrodes, whereas the output space is represented by $m$-dimensional activation values $\boldsymbol{\tau}$, to be fed to the robotic hand as (normalized) activation signals. The activation signals ranged between 0 and 1 , where 0 represents the condition of no current given to the motor and 1 the maximum current allowed by the motor. The activation signals were mapped directly to the PWM signal of the robotic hand, influencing the duty cycle, and consequently the current in each motor. Given an online training set, that is, a flow of (sEMG, activation) pairs, iRR incrementally computes an optimal linear model $W \in \mathbb{R}^{m \times d}$ such that $\boldsymbol{\tau}=W \boldsymbol{e}$. Since myocontrol at the level of single fingers cannot be linearly solved [7], we use RFFs to evaluate a non-linear mapping $\phi: \mathbb{R}^{d} \rightarrow \mathbb{R}^{D}$ and solve the linear regression problem in a feature space of dimension $D>d$, rather than directly in the input space. In particular, iRR with RFFs computes an optimal non-linear model $W^{\prime} \in \mathbb{R}^{m \times D}$ such that

$$
\boldsymbol{\tau}=W^{\prime} \phi(\boldsymbol{e}) .
$$

Since the feature space induced by $\phi$ is finite-dimensional (and its optimal dimension $D$ must be found by exhaustive search), the approach is strictly bounded in space, making it suitable for online learning. In addition, the optimal model $W^{\prime}$ can be easily evaluated by direct computation (no optimization algorithm is required) and can be updated at a reduced computational cost (exactly as it happens for iRR) using the selected rank-1 update method.

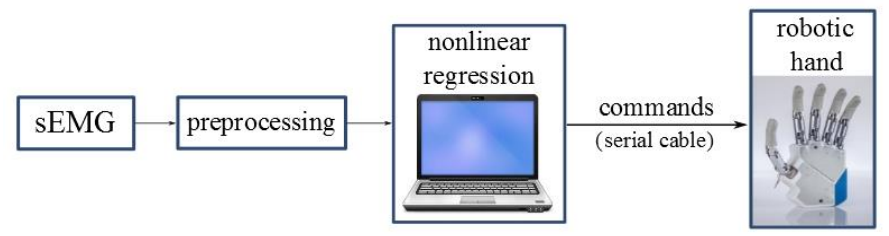

Fig. 1. Schematic concept of the system used to interface the sEMG signals to the control system embedded in the robotic hand. The preprocessing block represents the amplification, rectification and filtering performed in hardware by the Otto Bock EMG sensors used.

In this work the machine learning algorithm was trained by 
collecting, for each forearm, $d=10$ sEMG signals from the extrinsic muscles of the hand, associated to 5 grasps/postures; and by mapping those signals to $m=5$ activation vectors for each robotic hand. During training, only minimal and maximal activation values $\boldsymbol{\tau}$ were collected; nevertheless the predicted

TABLE I activations assumed graded values between 0 and 1 . This approach makes the training shorter, easier and more realistic [7]. The predicted $\boldsymbol{\tau}$ were fed online to the current control loop of the robotic hand.

ACTIVITIES OF DAILY LIVING USED IN THE FUNCTION TEST

\begin{tabular}{|c|c|c|c|c|c|}
\hline No. & Name & Description & $\begin{array}{l}\text { Single handed } \\
\text { /bimanual }\end{array}$ & $\begin{array}{l}\text { Hand configuration } \\
\text { required }\end{array}$ & Failure reasons \\
\hline $\mathrm{T} 1$ & Dial 1973 & Dial number 1-9-7-3 on the telephone pad & Single & Index pointing & Dial wrong number \\
\hline $\mathrm{T} 2$ & Pour water & Grasp a bottle and pour water in a mug & Single & Cylindrical & $\begin{array}{l}\text { Drop the bottle or place } \\
\text { it outside the target area }\end{array}$ \\
\hline $\mathrm{T} 3$ & Prism & Grasp and transport a prism to a target area & Single & Tridigital & $\begin{array}{l}\text { Drop the prism or place } \\
\text { it outside the target area }\end{array}$ \\
\hline $\mathrm{T} 4$ & Door handle & Turn a door handle & Single & Cylindrical & -- \\
\hline T5 & Credit card & Remove credit card from slot & Single & Lateral & Drop the credit card \\
\hline T6 & Tray & Grasp and transport a tray to a target area & Bimanual & Lateral/ lateral & $\begin{array}{l}\text { Drop the tray or place it } \\
\text { outside the target area }\end{array}$ \\
\hline $\mathrm{T} 7$ & $\begin{array}{l}\text { Unscrew jar } \\
\text { lid }\end{array}$ & $\begin{array}{l}\text { Grasp a jar with one hand and } \\
\text { unscrew the lid with the other hand }\end{array}$ & Bimanual & $\begin{array}{l}\text { Cylindrical/ tridigital } \\
\text { or } \\
\text { cylindrical/ cylindrical }\end{array}$ & $\begin{array}{l}\text { Drop the jar/jar lid or } \\
\text { place the jar outside the } \\
\text { target area }\end{array}$ \\
\hline $\mathrm{T} 8$ & Phone call & $\begin{array}{l}\text { Grasp a phone receiver with one hand and } \\
\text { dial } 1-9-7-3 \text { with the other hand }\end{array}$ & Bimanual & $\begin{array}{l}\text { Cylindrical/ index } \\
\text { pointing }\end{array}$ & $\begin{array}{l}\text { Dial wrong number or } \\
\text { drop the receiver }\end{array}$ \\
\hline T9 & Pass prism & Pass a prism from one hand to the other & Bimanual & Tridigital/ tridigital & $\begin{array}{l}\text { Drop the prism or place } \\
\text { it outside the target area }\end{array}$ \\
\hline $\mathrm{T} 10$ & Buffet & $\begin{array}{c}\text { Grasp a plate with one hand and } \\
\text { pick a ball from it with the other hand }\end{array}$ & Bimanual & Lateral/ tridigital & $\begin{array}{l}\text { Drop the plate } \\
\text { or the ball }\end{array}$ \\
\hline
\end{tabular}

Both the evaluation/update of $W^{\prime}$ and the prediction of $\boldsymbol{\tau}$ require a few milliseconds on a standard laptop, enabling a transparent integration with the rest of the setup, blurring the distinction between the training and the prediction phases, typical in machine learning systems. A concept schematic of the controller is shown in Fig. 1.

The EMG signals were collected from each forearm using 10 commercially available sEMG electrodes (model MyoBock 13E200, Otto Bock, Duderstadt, Germany). They process onboard the linear envelope of the raw EMG signal, thus providing an output signal which is correlated to the amplitude of the EMG signal of the muscles.

The sEMG signals were acquired at a rate of $12 \mathrm{~Hz}$, digitally filtered using a $1^{\text {st }}$ order low-pass Butterworth filter (cutoff frequency: $1.5 \mathrm{~Hz}$ ) and directly fed to the machine learning algorithm. At the same rate, the values $\boldsymbol{\tau}$ were predicted, lowpass filtered ( $1^{\text {st }}$ order Butterworth filter, cutoff frequency: $\left.1 \mathrm{~Hz}\right)$ and directly used to control the robotic hands.

\section{Experimental Protocol}

The ability of deploying the ILMC onto a robotic hand in order to carry out Activities of Daily Living was evaluated using a functional test inspired by the SHAP [16] (Fig. 2). Ten tasks were chosen (TABLE I) based on the approximate percentage of usage of the main grasps [19], which were the cylindrical, lateral, tridigital grasps and the index pointing (Fig. 2). Five of the ten tasks (T1..T5) required one hand to be performed whereas the other five (T6..T10) required bimanual manipulation. The subjects were asked to perform the single handed tasks (T1..T5) first with the right hand, then with the left hand, finally the bimanual tasks (T6..T10). Each task was performed in a series of five repetitions $\left(\mathrm{T} 1_{1} . . \mathrm{T} 1_{5}, \mathrm{~T} 2_{1} . . \mathrm{T} 2_{5}, \ldots\right)$, for a total of 75 tasks. The tasks were ordered with increasing difficulty of execution based on pilot tests, conducted by the expert subjects before the experimental session. In the pilot test each subject performed five repetitions of each task, measuring the proper Tcs. At the end of the pilot session the Tcs of the subjects were confronted and averaged and a list of tasks was redacted based on the decreasing speed of performance.

The execution time of each task was measured by each subject using a self-operated timer [16]. Two metrics were used to quantify the quality and performance of grasp: (1) the taskcompletion rate (CR), defined as the percentage of correctly executed tasks; (2) the task-completion time (Tc), defined as the time spent to perform the whole task as measured by the timer. A task was considered successful when none of the failure reasons occurred.

Before the experiment started each robotic hand was fixed onto an orthopedic splint which allowed reaching and grasping by able bodied subjects. The orthopedic splints were firmly fastened to the forearms of the subjects by means of three Velcro straps, to allow the subjects to perform only isometric contractions. The sEMG electrodes were applied on the subjects' forearms, placed uniformly around the forearm in the proximal region, about $10 \mathrm{~cm}$ below the olecranon, using an elastic band (Fig. 2). However, since an exact placement of the sEMG electrodes over the muscles was not necessary for the correct training of the ILMC system, the electrodes could be placed in almost in any position distally to the elbow as long as they covered as many different muscles as possible.

The four chosen grasps/postures (Fig. 2) were visually and verbally explained to the subjects, then the ILMC was trained 
with the muscular activity related to hand relax and to the grasps/postures (TABLE II). During the training phase the subjects were guided to perform the desired grasps/postures according to a visual cue on a computer screen. The hand relax was adopted as the rest configuration for the ILMC system (corresponding to hand relax in TABLE II), whereas the grasps/postures were performed by executing isometric contractions relative to the requested movement (cylindrical, tridigital, lateral, point finger): the subjects were instructed to use a medium/high force range during the maximum contraction for each grasp/posture, in order to avoid the effects of fatigue for the whole duration of the experimental session. Subjects performed this training phase with both hands, in two distinct arm configurations, in order to initialize correctly the first map of postures of the ILMC.

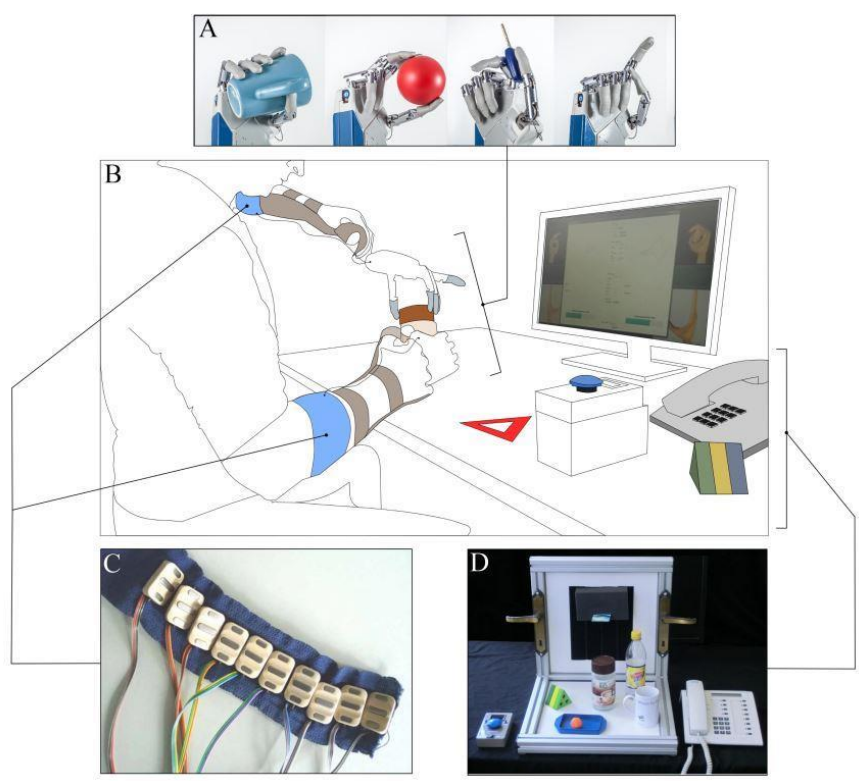

Fig. 2. A) The four grasps/postures used in the experiment: cylindrical, tridigital, lateral grasps and index pointing. B) Bimanual manipulation task: the subject is engaged in unscrewing the jar lid (T7 in TABLE I). C) 10 sEMG Ottobock electrodes set in an elastic band. D) The experimental setup consisted in a manually operated timer and ten objects of daily use.

The first configuration was performed with both arms close to the body and flexed elbows ( $\sim 90^{\circ} \mathrm{deg}$.), whereas the second was performed with both arms extended and forearms pronated; the sEMG signals differ consistently in the two configurations, adopting this strategy the regression algorithm could converge to the right grasp. When performing the training contractions for the four grasps/postures, the subjects were instructed to contract their muscles at roughly $40 \%$ maximal voluntary contraction. This value was used as the maximum activation level in the ILMC (associated to $\tau=1$ ) as it is known that comfortable contraction levels fall typically between $10 \%$ to $15 \%$ of maximum voluntary contraction [20].

After training the subjects were allowed to use the system for five to ten minutes in order to become familiar with it. Soon after the task series commenced. During the task series, the subjects were allowed to rest whenever desired, in order to make the experience as comfortable as possible. Every time a subject and/or the experimenters deemed that the predictor was not accurate enough, the system was retrained, only for the inaccurate grasps/postures. Such new data was added on-line to the current model, which was possible because of the ILMC approach. After retraining, the experiment would go on. No limitation was placed on the number of retraining phases. The retraining phase was enforced whenever the subject and/or the experimenters agreed that the task was too hard to be completed because of grasp instability, which did not necessarily lead to the "failure" of the task.

TABLE II

VALUES OF $\tau$ DURING The PREDiction Phase

\begin{tabular}{cccccc}
\hline \hline & $\begin{array}{c}\text { Abduction/ } \\
\text { Adduction }\end{array}$ & Thumb & Index & Middle & $\begin{array}{c}\text { Ring/ } \\
\text { Little }\end{array}$ \\
\hline Hand relax & 0 & 0 & 0 & 0 & 0 \\
Cylindrical & 1 & 1 & 1 & 1 & 1 \\
Tridigital & 1 & 1 & 1 & 1 & 0 \\
Lateral & 0 & 1 & 1 & 1 & 1 \\
Point & 1 & 1 & 0 & 1 & 1 \\
\hline \hline
\end{tabular}

We hypothesized a statistical difference between the two groups of subjects (naïve and experts) as measured by the completion times (Tc) of the 15 tasks and the means of the repetitions. To test this hypothesis a repeated measures 2-way ANOVA (ANOVA1) was calculated, to determine if there was a statistical difference between the Tcs of expert and naïve subjects. A second statistical analysis was performed afterwards to assess statistical differences within the groups, across subjects [factors: hand dominance (non-dominant and dominant hand) and the tasks (5 repetitions per task for each hand)]. Two repeated measures 2-way ANOVA tests were calculated, the first (ANOVA2) relative to the expert subjects (3 subjects) and the second (ANOVA3) relative to the naïve subjects (6 subjects). Lastly, four unpaired t-tests were calculated to highlight any significant difference in the number of retraining phases between naïve and expert subjects.

\section{RESULTS}

To illustrate the experiments, we provided one video clip as supplementary material, showing the subjects engaged in the experimental session.

The graphs in Fig. 3 show the acquired sEMG values of the right and left forearms associated with acquiring a representative task (i.e., T7 - Unscrew jar lid). The graphs also show the actual average predictions of the ILMC, the positions taken by the controlled DoAs and their motor currents. The duration of the window represents the completion time (Tc) of the third repetition of task T7 by one subject (S1). The hands were controlled in real-time during this task and the finger positions followed the prediction signals.

The completion rates (CR) resulted quite high for singlehanded tasks (right hand $90.7 \%$, left hand $95.6 \%$ ) and overall did not greatly vary across subjects and tasks (from $82 \%$ for T5 to $100 \%$ for T4) (Fig. 4). Tasks T1, T3, and T4 were performed with CR > 90\% with the right hand, whereas T1-T4 were performed with CR $>90 \%$ with the left hand. The $\mathrm{CR}$ was lower for bimanual tasks (83.6\%), with the lowest CR achieved in the case of the buffet task (66.7\%). 

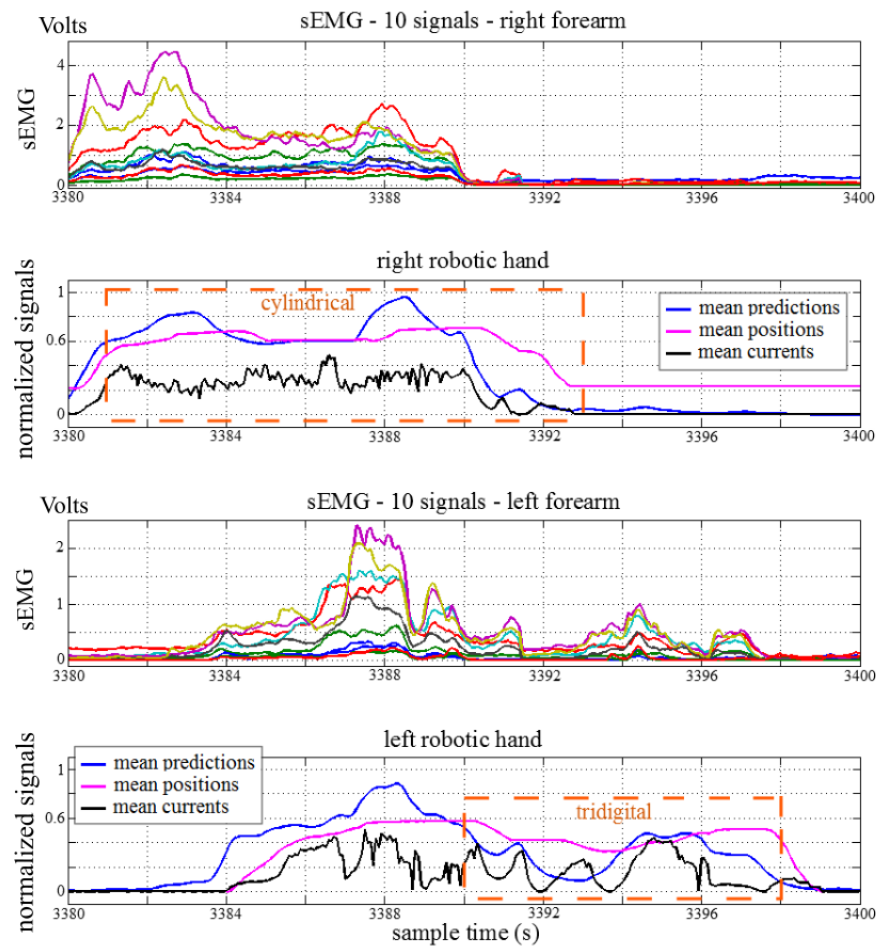

Fig. 3. Representative bimanual task - unscrew jar lid. Acquired sEMG signals (in Volts) vs. normalized predictions (output of the ILMC), finger positions and motor currents (output of the $\mathrm{IH} 2$ hands, averaged across DoAs) during task T7. The superimposed striped rectangles delimit the times when the proper grips were performed, in order to complete the task, i.e. cylindrical grasp with the right hand, and tridigital grasp with the left hand. The graph represents the full trial with $\mathrm{Tc}=20.12 \mathrm{~s}$. Mean predictions and mean positions are relative to the DoFs actuated during the current grasps, i.e. for the cylindrical grasp all the finger flexions are considered, for the tridigital grasp the three flexions of thumb, index and middle fingers are considered. In the graphs is evident the mean values of the fingers positions follow the mean values of the prediction signals.

The first two tasks of the experiment resulted with larger Tc for both hands when compared to the last three tasks (22.5 seconds vs. 11.5 seconds with the right hand; 15 seconds vs. 10.1 seconds with the left hand). The bimanual tasks were the most complex and difficult tasks, thus they required more time, as reflected from the results (Fig. 4). No clear learning curve appears within series.

If the completion time results are divided based on the familiarity with the system, some interesting insights could be gained (Fig. 5). The larger Tc seen for tasks 1 and 2 (Fig. 4) can be largely attributed to the naïve subjects which completed the tasks much slower than the expert ones. This was true for both hands. This difference in completion time could not be observed with the last three single-handed tasks. On the other hand the completion rates (CR) were similar for naïve and expert subjects and for this reason were not further analyzed.

The duration of the experiment, averaged across all subjects, was $96.62 \pm 22.05$ minutes (mean \pm standard deviation) of which $86.72 \pm 24.00$ minutes employed in the prediction phase, i.e. $\sim 10 \%$ of the total experiment time was spent in training $(\sim 5 \%$ of the time) or retraining ( $\sim 5 \%$ of the time) the ILMC. On average $7 \pm 4.5$ (median \pm semi-interquartile range) retraining sessions were required by each subject during the experiment, each one lasting $39.14 \pm 17.16$ seconds $(0.75 \%$ the total average prediction time). The proportion of initial training, prediction and retraining time remained uniform across all subjects, where the prediction phase occupied the vast majority of the total time.

Considering the distribution of the retraining sessions across the nine experiments (Fig. 6), no evident pattern emerges. On average, after the initial training session, the four grasps/postures were retrained uniformly: in total $2 \pm 0.5$ (medians \pm semi-interquartile ranges), $1 \pm 2,3 \pm 0.5$ and $1 \pm 0.5$ retraining sessions were required for the cylindrical, tridigital, lateral grasps and index pointing, respectively (Fig. 7).
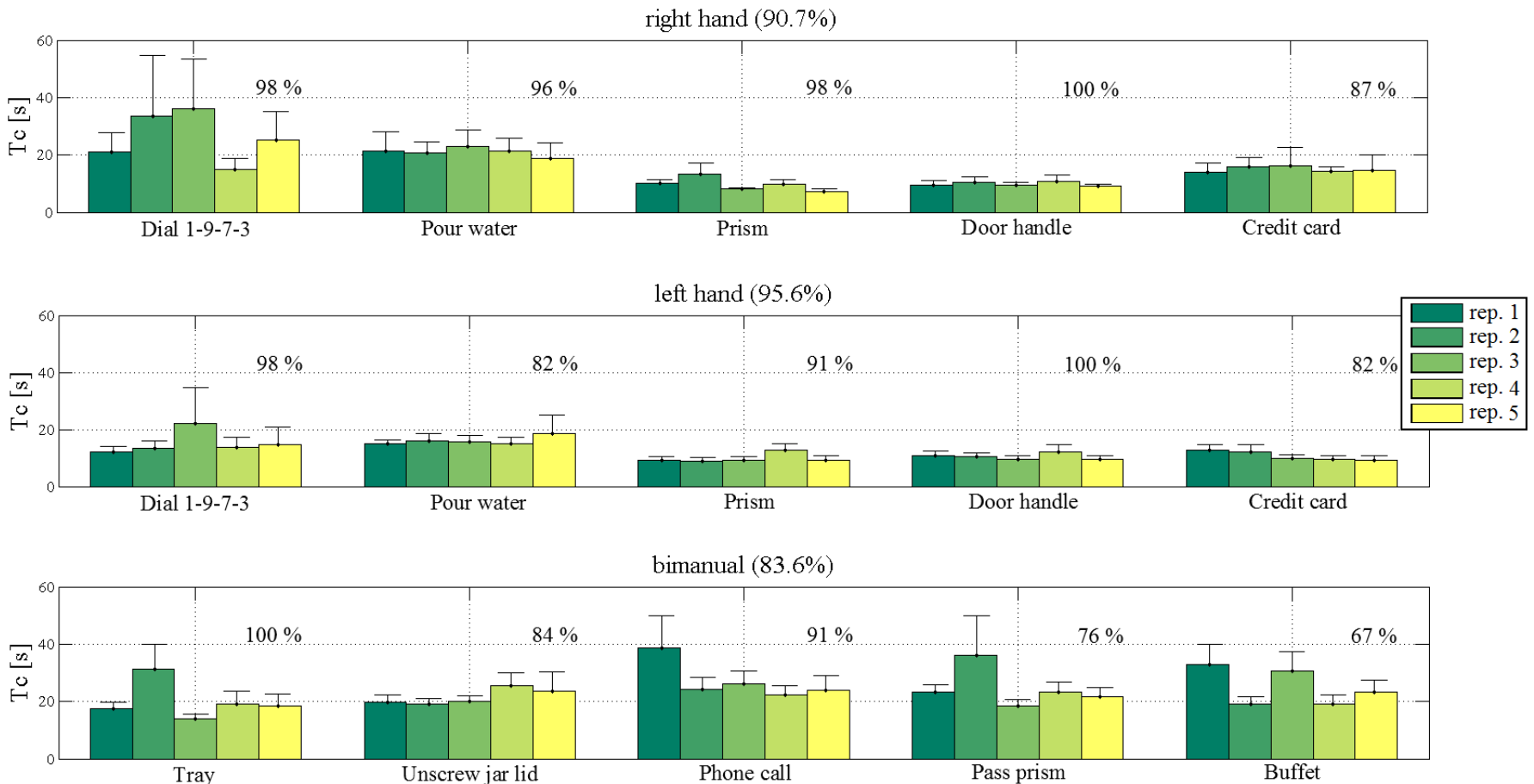

Fig. 4. Results. Completion times (Tc), on the y axis, and completion rates (CR, superimposed on the graphs) achieved during the experiment. Mean \pm SEM (standard error of the mean, represented by the error bars) calculated across all the nine subjects. Task repetitions are showed using different color bars. 
In total, during the entire experimental session, the retraining phase were needed 80 times (in turn 21, 26, 20 and 13 times for the cylindrical, tridigital, lateral grasps and index pointing); in 57 of these cases no further retraining phases were needed for the same pattern immediately after, and the task could be successfully completed. This means that, in $71.25 \%$ of the cases, the retraining of one single pattern (associated to one grasp/posture) enabled the subject to carry on with the experiment.
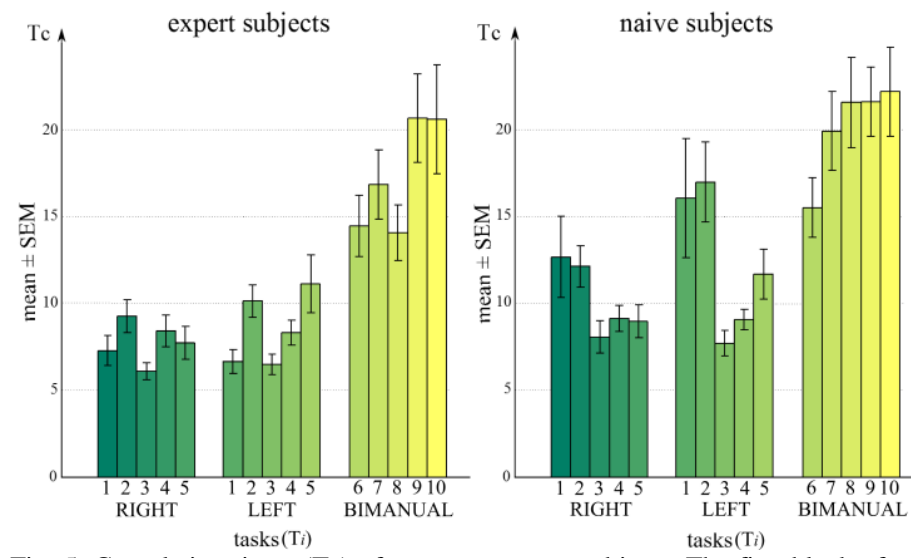

Fig. 5. Completion times (Tc) of expert vs. naïve subjects. The first block of bars refers to the right hand tasks, the second block to the left hand tasks and the third block to the bimanual tasks. Mean \pm SEM of Tcs of all repetitions of the same task calculated separately for expert and naïve subjects. Completion rates $(\mathrm{CR})$ values are not shown.

The statistical analysis relative to the ANOVA1 revealed a high difference between the two groups $(F(1,134)=24.25$, $p<0.001$ ), as expected from the previous analysis and the observation of Fig. 5. This result was expected due to the different capability of expert and naïve subjects to execute naturally the requested tasks.

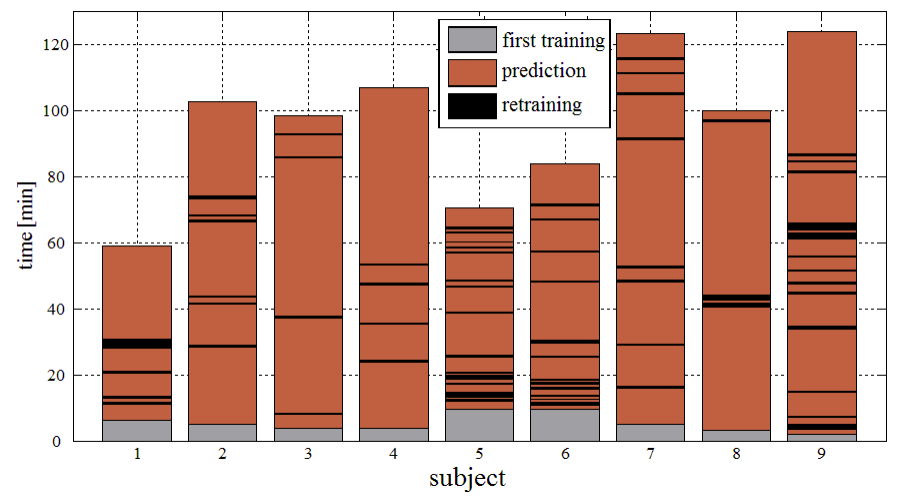

Fig. 6. Durations and temporal synchronization of the experimental phases per subject. The black lines denote the exact time of retraining during the experiment.

The ANOVA2 analysis results did not reveal a significant statistical difference neither for the hand dominance $(F(1,128)=0.76, p=0.47)$ nor for the tasks $(F(4,128)=2.80$, $p=0.10)$; also the results for the interactions between the factors did not show a significant statistical difference. Similarly the ANOVA3 results did not reveal any significant statistical difference for the hand dominance $(F(1,260)=0.35, p=0.58)$ nor for the tasks $(F(4,260)=0.10, p=0.98)$. The results for the interactions between the factors showed a significant statistical difference $(p<0.05)$ for the hand dominance with tasks $(F(5,260)=2.31, p=0.04)$.

Lastly, the results of the four unpaired t-tests showed no significant statistical difference between expert and naïve subjects in the number of retraining phases, calculated for each grasp/posture performed (cylindrical: $p=0.68$; tridigital: $p=0.23$; lateral: $p=0.08$; point: $p=0.16$ ).

The results of the statistical analysis were expected because the system implemented to conduct this experiment was symmetrical (no influence of hand dominance) and the ILMC control system was realized to make the subject comfortable to use it both with their dominant or non-dominant hand.

These results confirm our hypotheses that the ILMC system was easy and very intuitive to use, both in the situations of nondominant hand and/or of naïve users.

The statistical analysis of the completion rates (CRs) was not conducted since the values of CRs were similar for naïve and expert subjects and for this reason were not further analyzed.

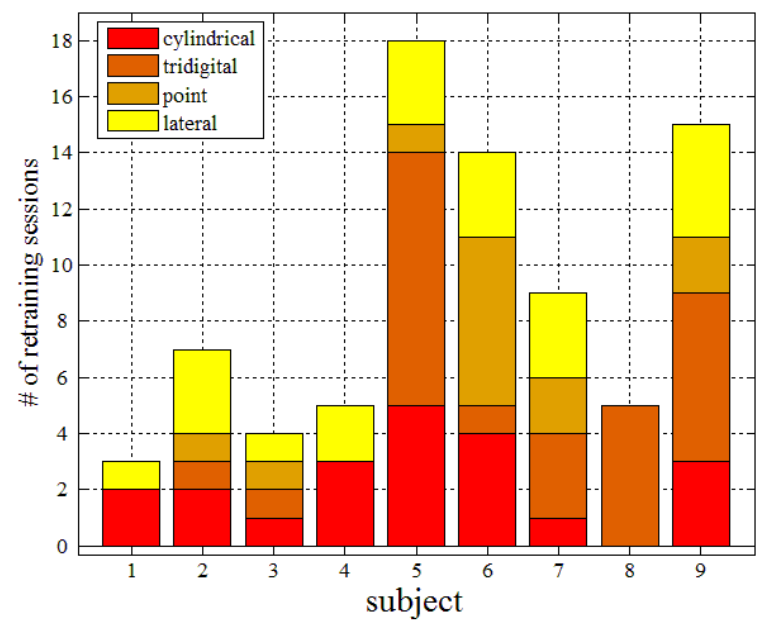

Fig. 7. Required retraining sessions during the functional test per subject.

\section{DISCUSSION}

In this paper we have demonstrated the applicability of iRR with RFFs, an Incremental-Learning Myoelectric Control (ILMC) system introduced in [7], to a bimanual manipulation task performed by nine able-bodied subjects. This is, to the best of our knowledge, the first demonstration of this kind. The incrementality of the ILMC is in this case exploited to update the myocontrol model (retraining phases) whenever required by the subject, due to, e.g., a change in the signal, electrode displacement, fatigue, etc. It represents a shift of focus from the calibration of a learning machine to reciprocal learning, in which a dissatisfied user can learn to better use the prosthesis as the model adapts to different conditions. The duration of the retraining phase is relatively short and this is due to the mathematical and implementative structure of the ILMC system: the update of the internal parameters is complete after one retraining of the strength patterns relative to the grasp/postures (TABLE II). 
The videoclip attached as supplementary material shows, in fact, some of the "tricks" enforced by the subjects during the execution of the more complex tasks; for example, shifting an object which is found in the way, or keeping an object still with one hand while operating on it with the engaged one. During the exemplary execution of task T7 ("unscrew jar lid", from 2:30 on) in particular, the power grasp enforced by the right hand is clearly unstable; nevertheless the subject succeeds in performing it, although it requires three successive attempts.

On-demand model update can counter the adverse, destabilizing effects of the changes in the sEMG signals and in the subject's posture, thus dramatically improving the reliability of the control system. Since this is the major reason for hand prosthesis abandonment [21] we claim that the concept of ILMC represents a major advancement with respect to the standard "pattern-recognition" myocontrol. In this particular experiment, in the vast majority of retraining sessions, retraining only once sufficed to enable the subject to carry on with the experiment, thus validating the reliability of the ILMC control system.

Further research is required to assess this improvement, possibly by conducting the same experiment with and without the ILMC, on amputated subjects; so far, our experiments were rather aimed at assessing user performance in using the ILMC with two robotic hands. The results we have shown confirm the subjects' learning capabilities: at the end of the experiments both naïve and expert subjects reached practically the same performance in terms of completion rate (CR) and completion time (Tc). We argue that this is due, besides the usage of an ILMC, to the flexibility offered by the control system, that does not implement a stereotypical grasp, but allows a graded control of the motor activations by the subject, in a really intuitive manner, so that he/she can find his/her own personal way to perform a grasp. This position was confirmed by the statistical analysis presented, where the same statistical result was found for the groups of naïve users and expert ones: no statistical difference with respect to the hand dominance or to the task executed.

Bimanual tasks had lower CRs with respect to single-handed tasks; this seems reasonable since the former were more complex to perform, requiring the coordination of reaching and grasping of the two robotic hands. The lowest CR of all $(66.7 \%)$ was achieved with the buffet task, requiring accurate, coordinated and graded grasping with both hands (i.e., lateral and tridigital). This task was probably the most difficult one to execute with the present robotic setup [22].

Increased values of Tc for the naïve subjects were observed for tasks T1 and T2 (both left- and right-handed, Fig. 5). It is reasonable to claim that the naïve subjects needed more time in order to learn how to operate the ILMC system, before being able to use it comparably with expert users. This seems to be confirmed by the fact that the completion times of naive subjects during bimanual tasks are not statistically significantly different from those of the experts (Fig. 4, Fig. 5).

From the completion times data (Fig. 4) it is evident that subjects S7 and S9 had globally the worst performance; in particular, during the experiment S9 was not able to perform any lateral grasp, although several retrains were done to improve the subject's performance.

Although it seems from Fig. 6 that the retraining had to be performed quite often through the session, during the pilot test and the experimental session, it was noticed that a continuative use of the system improves the ability of the subject to learn how to initialize correctly the first map of postures of the ILMC during the training phase (TABLE II). This was confirmed by the statistical analysis that showed a significant difference between expert and naïve subjects' performances in terms of Tcs. This difference of Tcs is mainly attributed to the inexperience of the naïve subjects using the ILMC system and particularly during the initialization of the map of postures. For these reasons the continuative usage of the ILMC will avoid frequent retrainings of the system, that are a result of further modifications of the muscles movements during the grasps/postures. The facility of controlling the prosthetic hands movements using the ILMC permits every user to learn easily how to work with the system and rapidly improve manipulation performances. The lack of statistical difference between the number of retraining phases between naïve and expert subjects can be due to the ease of use of the ILMC, which was intuitive to control even for naïve subjects.

A final remark to be considered in the next study is to increase the number of participants in order to improve the robustness of the statistical analysis.

As a general, final remark, except for S9, every subject was able to perform each task (single-hand and bimanual) with no particular difficulties; for this reason, we claim that the ILMC was easy to use, as we wanted to prove at the beginning of this work.

To sum up, we believe that the usage of an incremental machine learning method in myocontrol paves the way to a more stable and reliable control of prosthetic hands. iRR with RFFs is one such type of control.

\section{ACKNOWLEDGMENT}

This work is partially funded by the EU Projects WAY (FP7288551) and THE (FP7-248587).

\section{REFERENCES}

[1] F. R. Finley and R. W. Wirta (1967). Myocoder studies of multiple myopotential response. Archives of Physical Medicine and Rehabilitation. 11(48), 598-601.

[2] A. H. Bottomley (1965). Myoelectric control of powered prostheses. J Bone Joint Surg B. 47:411-5.

[3] D. J. Atkins, D. C. Heard, and W. H. Donovan (1996). Epidemiologic overview of individuals with upper-limb loss and their reported research priorities. JPO: Journal of Prosthetics and Orthotics, 8(1), $2-11$.

[4] P. J. Kyberd, C. Wartenberg, L. Sandsjö, S. Jönsson, D. Gow, J. Frid and L. Sperling (2007). Survey of upper-extremity prosthesis users in Sweden and the United Kingdom. JPO: Journal of Prosthetics and Orthotics, 19(2), 55-62.

[5] N. Jiang, T. Lorrain, and D. Farina. (2014). A state-based, proportional myoelectric control method: online validation and comparison with the clinical state-of-the-art. Journal of neuroengineering and rehabilitation, 11(1), 110.

[6] P. Parker, K. Englehart and B. Hudgins (2006). Myoelectric signal processing for control of powered limb prostheses. Journal of electromyography and kinesiology, 16(6), 541-548. 
[7] A. Gijsberts, R. Bohra, D. Sierra González, A. Werner, M. Nowak, B. Caputo, M. Roa and C. Castellini (2014). Stable myoelectric control of a hand prosthesis using non-linear incremental learning. Frontiers in Neurorobotics $8(8)$.

[8] A. E. Hoerl and R. W. Kennard (1970). Ridge Regression: Biased Estimation for Nonorthogonal Problems. Technometrics 12, 55-67.

[9] A. Rahimi and B. Recht (2008b). Uniform approximation of functions with random bases. Allerton Conference on Communication Control and Computing (Allerton08) (UrbanaChampaign,IL), 555-561. doi: 10.1109/ALLERTON.2008.4797607

[10] N. Jiang, K. B. Englehart and P. A. Parker (2009). Extracting Simultaneous and Proportional Neural Control Information for Multiple-DOF Prostheses From the Surface Electromyographic Signal. IEEE Transactions on Biomedical Engineering 56(4), 10701080.

[11] S. Muceli and D. Farina. Simultaneous and proportional estimation of hand kinematics from EMG during mirrored movements at multiple degrees-of-freedom. Neural Systems and Rehabilitation Engineering, IEEE Transactions on 20.3 (2012): 371-378.

[12] A. Ameri, E. Kamavuako, E. Scheme, K. Englehart, P. Parker, "Support Vector Regression for Improved Real-Time, Simultaneous Myoelectric Control," IEEE Transactions on Neural Systems and Rehabilitation Engineering, to appear.

[13] A. J. Young, L. H. Smith, E. J. Rouse and L. J. Hargrove (2013). Classification of simultaneous movements using surface EMG pattern recognition. Biomedical Engineering, IEEE Transactions on, 60(5), 1250-1258.

[14] J.W. Sensinger, B.A. Lock, and T.A. Kuiken (2009). Adaptive pattern recognition of myoelectric signals: exploration of conceptual framework and practical algorithms. Neural Systems and Rehabilitation Engineering, IEEE Transactions on, 17(3), pp.270278.

[15] J.M. Hahne, S. Dahne, H.J. Hwang, K.R. Muller and L.C. Parra (2015). Concurrent adaptation of human and machine improves simultaneous and proportional myoelectric control. Neural Systems and Rehabilitation Engineering, IEEE Transactions on, 23(4), pp.618-627.

[16] C.M. Light, P.H. Chappell, P.J. Kyberd (2002, June). Establishing a Standardized Clinical Assessment Tool of Pathologic and Prosthetic Hand Function: Normative Data, Reliability, and Validity. Archive of Physical Medicine and Rehabilitation, vol. 83.

[17] L.M. Hermansson, A.G. Fisher, B. Bernspång and A.C. Eliasson (2005). Assessment of capacity for myoelectric control: a new Raschbuilt measure of prosthetic hand control. Journal of rehabilitation medicine, 37(3), pp.166-71.

[18] A. Rahimi and B. Recht (2008). Random Features for Large-Scale Kernel Machines, Advances in Neural Information Processing Systems 20, 1177-1184.

[19] C. Cipriani, M. Controzzi and M. C. Carrozza (2011). The SmartHand transradial prosthesis. Journal of neuroengineering and rehabilitation, 8(1), 29.

[20] W. Rohmert, "Ermittlung von Erholungspausen für statische Arbeit des Menschen" Internationale Zeitschrift für angewandte Physiologie einschließlich Arbeitsphysiologie, vol. 18, no. 2, pp. 123$164,1960$.

[21] B. Peerdeman, D. Boere, H. Witteveen, R. Huis in 't Veld, H. Hermens, S. Stramigioli, H. Rietman, P. Veltink and S Misra (2011). Myoelectric forearm prostheses: State of the art from a user-centered perspective, Journal of Rehabilitation Research and Development 6(48), 719-738.

[22] G. C. Matrone, C. Cipriani, E. L. Secco, G. Magenes and M. C. Carrozza (2010). Principal components analysis based control of a multi-dof underactuated prosthetic hand. Journal of neuroengineering and rehabilitation, 7(16), 1-16. 